\title{
Metastases in axillary sentinel lymph nodes in breast cancer as detected by intensive histopathological work up
}

\author{
Gábor Cserni
}

\begin{abstract}
Aim-To assess the value of the intensive histological work up of axillary sentinel lymph nodes (SLN) to demonstrate regional metastatic disease.

Methods-From a series of 58 successful lymphatic mapping procedures, 78 SLN were analysed by serial sections (mean of 49 levels/SLN) and by immunostaining to cytokeratin and epithelial membrane antigen, and the results compared with those obtained by assessing the central cross section.

Results-The central cross section would have failed to detect metastases in eight of 26 lymph nodes (31\%) in patients with breast cancer metastasising to the SLN only. This would have led to a false negative node status in six of 21 patients $(29 \%)$. Two micrometastases were detected with the aid of immunostains.

Conclusions-The results suggest the need to examine SLN at multiple levels and to use immunohistochemistry in negative cases. Serial sections are also useful in the case of micrometastases, as some of these may convert to macrometastases at deeper levels. Multiple level investigation of SLN and immunohistochemistry in the event of the negativity of standard stains would result in improved staging and an increase in the proportion of node positive disease detected.

(F Clin Pathol 1999;52:922-924)
\end{abstract}

Keywords: axillary sentinel nodes; serial sectioning; immunohistochemistry

The sentinel lymph node (SLN) concept has been studied in various contexts, including penile carcinoma, ${ }^{1}$ malignant melanoma of the skin, ${ }^{2}$ breast cancer, ${ }^{34}$ and recently thyroid neoplasms. ${ }^{5}$ Oral cancers and colorectal cancers have also been investigated. More and more data are accumulating to suggest that the SLN is the first target of lymphogenic metastasis in these tumours. The aim of the present study was to investigate the value of serial sectioning and immunostaining of SLN for an assessment of the overall axillary nodal status of breast cancer patients.

\section{Methods}

Most tumours reported here were diagnosed as malignant by fine needle aspiration (FNA) cytology preoperatively, except for 10 , where intraoperative frozen sections were performed because of equivocal or missing FNA results. From 70 lymphatic mapping procedures involving the use of peritumorally injected patent blue dye, SLN were successfully identified in 58 tumours: one in situ ductal carcinoma, and one microinvasive, $18 \mathrm{pT} 1$, and $38 \mathrm{pT} 2$ breast cancers. There were six tubular mixed, three tubular, three lobular, one mucinous, and one ductal and lobular mixed special type carcinomas. All the remaining tumours were no special type ductal carcinomas. In all, 78 SLN (mean 1.3 , range 1 to 3) were recovered, with a mean of 19 non-SLN per patient (range 7 to 42 ). The SLN were removed separately before completion of axillary dissection. Further technical details of the mapping procedure have been reported elsewhere. ${ }^{6}$

All SLN were fixed in 7\% buffered formalin. Smaller nodes were embedded in paraffin in toto, while larger nodes were bisected, with both halves being processed. All the SLN were sectioned serially up to extinction of the blocks, except for those of five patients in whom there was massive involvement macroscopically. Not all 3-5 $\mu \mathrm{m}$ thick levels were taken for histology, but every 10 th to 20 th level was examined and, for a given node, the depths between the examined sections were approximately the same. After every 6th section taken for haematoxylin and eosin $(\mathrm{H} \& \mathrm{E})$ staining, one was taken for cytokeratin (Novocastra, NCL-PAN-CK, 1:100 dilution) and epithelial membrane antigen (EMA Biogenex, MU-182-UC, 1:50 dilution), alternately. These latter were investigated only in the case of negative $\mathrm{H} \& \mathrm{E}$ findings. The third dimension of the SLN was then reconstructed on the basis of serially numbered sections.

\section{Results}

Of the total of 39 node positive patients, judged on the basis of all nodes, 36 were also SLN positive; in 21 patients the SLN were the only nodes with metastasis, with five instances of micrometastasis (N1a as defined by the American Joint Committee on Cancer; metastases not larger than $2 \mathrm{~mm}){ }^{7}$ The SLN involvement of these 21 patients is shown in fig 1 .

With the exclusion of SLN Nos 8 and 13b, which were macroscopically metastatic and cut at only nine and three levels, respectively, the SLN were investigated by means of $\mathrm{H} \& \mathrm{E}$ staining at a mean of 49 levels (range 25 to 102). It can be seen from fig 1 that one or two close levels from the central area of the SLN would have failed to detect metastases in eight of the 26 SLN (31\%), and this would have led
Accepted for publication 24 May 1999 


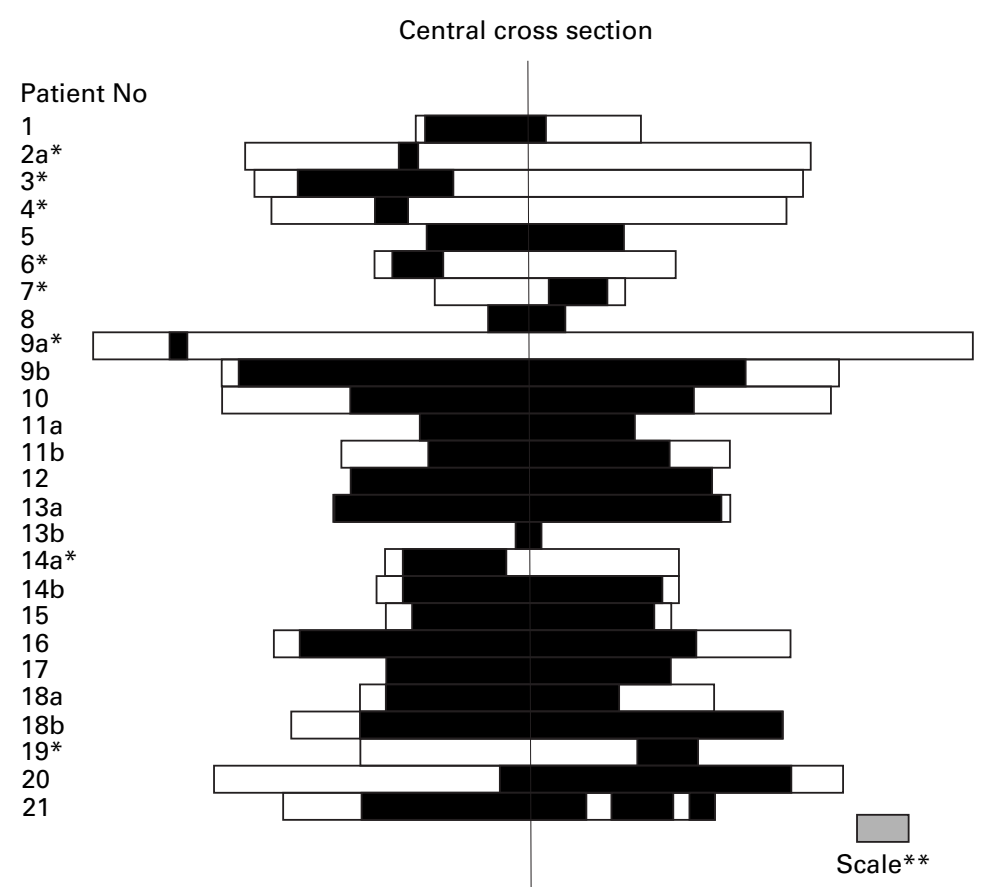

Figure 1 Distribution of metastases in sentinel lymph nodes (SLN) that were associated with non-involved non-SLN in relation to the central cross section. The asterix denotes

SLN in which the metastasis would have been missed if only the central cross section area had been assessed. Multiple metastatic SLN from the same patient are distinguished with letters. Black, areas with metastasis; white, areas without metastasis. ${ }^{\star *}$ The scale represents five equidistant levels at 50-100 $\mu \mathrm{m}$ distance from each other, depending on overall nodal size.

to the assignment of a false negative node status to six of the 21 patients $(29 \%)$ with SLN located metastases only.

In two cases (both ductal carcinomas of no special type), micrometastases were seen on $\mathrm{H} \& \mathrm{E}$ slides only after the immunostains had drawn attention to them. One of these (patient No 2; PAN-CK positive only) is included in fig 1 ; the other (EMA positive only) had metastases in three non-sentinel nodes as well. Immunostains were of no help in identifying further metastases.

\section{Discussion}

Because of the relatively high cost of gamma probes, the lymphatic mapping procedure involved in this study used a vital blue dye technique. Technetium-99m labelled colloids and gamma probes have been increasingly used for the identification of SLN, and the combination of the two techniques seems to give the highest success rates. ${ }^{8}$ However, vital blue dyes alone may give reliable results. ${ }^{9} 10$ Our series suggests that serial sectioning or immunostaining of the SLN would lead to better staging and would result in the conversion of six of 21 patients $(29 \%)$ considered node negative on the basis of routinely processed SLN. These techniques are not cost-effective as concerns all nodes, but the costs seem to be reasonable for a limited number of SLN.

A pilot study comparing the routine (one central cross section) work up of SLN and subsequent serial sectioning and immunostaining to cytokeratin with CAM 5.2 revealed a $23 \%$ conversion rate (three of 13 ) in originally SLN negative patients. ${ }^{11}$ Giuliano and col- leagues also compared their SLN biopsy and axillary dissection results (with special processing of SLN) with those gained by axillary dissection and routine work up of lymph nodes. ${ }^{12}$ They found a statistically significant increase in node positive cases $(38.2 \% v$ $29.1 \%$ ) which could be explained by the more intensive investigation of the SLN. The significance was even higher for micrometastatic disease $(3 \% v 16 \%)$. These data are somewhat different as regards absolute values, but seem similar in tendency to our own experience. Because of the lack of an organised screening programme, the median tumour size is larger $(2.5 \mathrm{~cm})$, and the proportion of node negative cases is $40-50 \%$. With the introduction of sentinel lymphadenectomies and the special work up of these nodes, nodal positivity has risen to $62 \%$. The frequency of diseases considered to be micrometastatic on the basis of the central cross section generally used was $1-2 \%$ during the previous five years, but has become $12 \%$ (seven of 58; two micrometastases in nonsentinel nodes) during the one year period of the series analysed.

The extent of histopathological work up remains controversial. Many investigators find routine processing enough, ${ }^{813-21}$ while others use special techniques, such as serial sectioning and immunohistochemistry. ${ }^{4} 10$ 22-24 Jannink and colleagues worked with a mean 14 sections per node and immunohistochemistry, ${ }^{11}$ while Giuliano's group used three levels in bivalved nodes, including immunostained slides. ${ }^{25}$ Our approach is certainly not to be recommended as a routine procedure for all SLN, because it is too labour intensive and costly. Before the era of immunohistochemistry, Pickren proposed a three level approach for all nodes ${ }^{26}$ but the histopathologically validated SLN theory ${ }^{25}$ could indicate that only the SLN should be investigated with this technique. This would result in reduced costs. The adequacy of investigating three levels at approximately $25 \%, 50 \%$, and $75 \%$ of the tissue blocks has recently been substantiated by finding only one micrometastasis with further serial sectioning and cytokeratin immunohistochemistry in $707 \mathrm{lymph}$ nodes from 34 patients considered node negative on the basis of the three levels originally investigated. Even this micrometastasis was found in one of the two patients whose nodes were originally analysed at the central cross section only. ${ }^{27}$ On the other hand our results suggest that a three level approach would have missed four of the metastases (15\%) in SLN Nos 2a, 6, $9 \mathrm{a}$, and 19, and would have resulted in downstaging three patients $(14 \%)$. All but one of these four metastases were micrometastases. Thus more than three levels should be investigated with $\mathrm{H} \& \mathrm{E}$ and immunostains in the event of the negativity of the SLN, but a three level approach may be sufficient and advisable as a first investigation. (Taking up to four separate blocks from each node, depending on overall nodal size, has also been recommended by the United Kingdom NHS breast screening programme.) Further serial (or step) sectioning would also be necessary for micrometastases, as these might become larger at deeper 


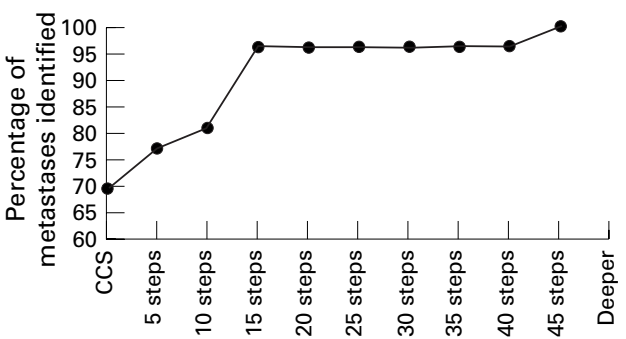

Figure 2 The number of step sections required for the identification of a given percentage of metastases limited to the sentinel lymph nodes. The steps range between 50 and $100 \mu \mathrm{m}$ depending on overall node size. CCS, central cross section; $100 \%=26$.

levels. Figure 2 gives an estimate for the number of steps to be sectioned for revealing all metastatic deposits.

We feel there are increasing data supporting the need for an intensive histopathological work up of SLN and the resulting improved staging. Although there is no consensus about the significance of finding micrometastatic deposits in loco-regional lymph nodes, a recent review has concluded that even micrometastases represent a survival disadvantage ${ }^{28} \mathrm{How}-$ ever, such a disadvantage can be demonstrated only in studies that involve enough patients and a substantial follow up period. The presence of micrometastases would indicate adjuvant systemic treatment in most clinical settings. SLN involvement detected by the more sensitive reverse transcription polymerase chain reaction will also require survival studies. ${ }^{29}$ However, care must be taken when subgroup analyses of patient survival are performed, and the Will Rogers statistical artefact of stage migration should be considered seriously. ${ }^{30}$ A final consideration is that, although the SLN theory is supported by increasing amounts of data, adequate prospective studies need to be performed to clarify the clinical relevance of improved staging resulting from a more detailed histopathological or molecular work up of SLN.

This work was supported by a János Bolyai Research Fellowship of the Hungarian Academy of Sciences. The author also acknowledges the help of Drs G Boross and B Baltás for providing him with the sentinel nodes and clinical information.

1 Cabanas RM. An approach for the treatment of penile carcinoma. Cancer 1977;39:456-66.

2 Morton DL, Wen DR, Wong J, et al. Technical details of intraoperative lymphatic mapping for early stage intraoperative lymphatic mapping

3 Krag DN, Weaver DL, Alex JC, et al. Surgical resection and Krag DN, Weaver DL, Alex JC, et al. Surgical resection and
radiolocalization of sentinel lymph node in breast cancer radiolocalization of sentinel lymph node in breast

4 Giuliano AE, Kirgan DM, Guenther JM, et al. Lymphatic Giuliano AE, Kirgan DM, Guenther JM, et al. Lymphatic
mapping and sentinel lymphadenectomy for breast cancer. mapping and sentinel lymp

5 Kelemen PR, Van Herle AJ, Giuliano AE. Sentinel lympadenectomy in thyroid malignant neoplasms. Arch Surg 1998;133:288-92.
6 Cserni G, Boross G, Baltás B. The value of axillary sentinel nodal status in breast cancer. World $\mathcal{F}$ Surg (in press).

7 American Joint Committee on Cancer. Breast. In: Fleming ID, Cooper JS, Henson DE, et al, eds. AfCC cancer staging manual, 5th ed. Philadelphia: Lippincott-Raven, 1997:17180 .

8 Albertini JJ, Lyman GH, Cox C, et al. Lymphatic mapping and sentinel node biopsy in the patient with breast cancer. ҰAMA 1996;276:1818-22.

9 Giuliano AE, Jones RC, Brennan $\mathrm{M}$, et al. Sentinel lymphadenectomy in breast cancer. 7 Clin Oncol 1997; 15: 2345-50.

10 Guenther JM, Krishnamoorthy M, Tan LR. Sentinel ymphadenectomy for breast cancer in a community managed care setting. Cancer F Sci Am 1997;3:336-40.

11 Jannink I, Fan M, Nagy S, et al. Serial sectioning of sentinel nodes in patients with breast cancer: a pilot study. Ann Surg Oncol 1998;5:310-14.

12 Giuliano AE, Dale PS, Turner RR, et al. Improved axillary staging of breast cancer with sentinel lymphadenectomy. Ann Surg 1995;180:700-4.

13 Roumen RM, Valkenburg JG, Geuskens LM. Lymphoscintigraphy and feasibility of sentinel node biopsy in 83 patients with primary breast cancer. Eur F Surg Oncol 1997; 23:495-502.

14 Dale PS, Williams JT. Axillary staging utilizing selective sentinel lymphadenectomy for patients with invasive breast carcinoma. Am Surg 1998;64:28-32.

15 Barnwell JM, Arredondo MA, Kollmorgen D, et al. Sentinel node biopsy in breast cancer. Ann Surg Oncol 1998;5:12630

16 O’Hea BJ, Hill AD, El-Shirbiny AM, et al. Sentinel lymph node biopsy in breast cancer: initial experience at Memorial Sloan-Kettering Cancer Center. 7 Am Coll Surg 1998;186:423-7.

17 Reuhl T, Kaisers H, Markwardt J, et al. Axillaausraumung bei klinisch nodal-negativen Mammakarzinom. Kann die Indikation durch "sentinel node"-Nachweis individualisiert werden? Dtsch Med Wochenschr 1998;123:583-7.

18 Koller M, Barsuk D, Zippel D, et al. Sentinel lymph node nvolvement-a predictor for axillary node status with breast cancer-has the time come? Eur F Surg Oncol 1998; 24:166-8.

19 Miner TJ, Shriver CD, Jaques DP, et al. Ultrasonographically guided injection improves localization of the radiolabeled sentinel lymph node in breast cancer. Ann Surg Oncol 1998;5:315-21.

20 Krag DN, Ashikaga T, Harlow SP, et al. Development of sentinel node targeting technique in breast cancer patients. Breast F 1998;4:67-74.

21 Flett MM, Going JJ, Stanton FD, et al. Sentinel node localization in patients with breast cancer. Br F Surg 1998;85: 991-3.

22 Pijpers R, Meijer S, Hoekstra OS, et al. Impact of lymphoscintigraphy on sentinel node identification with technetium-99m-colloidal albumin in breast cancer. $7 \mathrm{Nucl}$ Med 1997;38:366-8.

23 Veronesi U, Paganelli G, Galimberti V, et al. Sentinel-node biopsy to avoid axillary dissection in breast cancer with clinically negative lymph-nodes. Lancet 1997;349:1864-7.

24 Borgstein PJ, Pijpers R, Comans EF, et al. Sentinel lymph node biopsy in breast cancer: guidelines and pitfalls of lymphoscintigraphy and gamma probe detection. $f \mathrm{Am}$ Coll Surg 1998;186:275-83.

25 Turner RR, Ollila DW, Krasne DL, et al. Histopathologic validation of the sentinel lymph node hypothesis for breast carcinoma. Ann Surg 1997;226:271-6.

26 Pickren JW. Significance of occult metastases-a study of breast cancer. Cancer 1961;14:1266-71.

27 Zhang PJ, Reisner RM, Nangia R, et al. Effectiveness of multiple-level sectioning in detecting axillary nodal micrometastasis in breast cancer. A retrospective study with immunohistochemical analysis. Arch Pathol Lab Med 1998; 122:687-90.

28 Dowlatshahi K, Fan M, Snider HC, et al. Lymph node micrometastases from breast carcinoma. Reviewing the dilemma. Cancer 1997;80:1188-97.

29 Bostick PJ, Huynh KT, Sarantou T, et al. Detection of metastases in sentinel lymph nodes of breast cancer patients by multiple-marker RT-PCR. Int $f$ Cancer 1998;79:645-51.

30 Feinstein AR, Sosin DM, Wells CK. The Will Rogers phenomenon. Stage migration and new diagnostic techniques as a source of misleading statistics for survival in cancer. N Engl f Med 1985;312:1604-8. 